\title{
Selective Inhibition of Spindle Microtubules by a Tubulin-Binding Quinazoline Derivative ${ }^{\mathrm{S}}$
}

\author{
Jun-ichi Sawada, Hirosuke Ishii, Kenji Matsuno, ${ }^{3}$ Masayuki Sato, Yumiko Suzuki, ${ }^{2,4}$ \\ and Akira Asai ${ }^{1}$ \\ Center for Drug Discovery, Graduate School of Pharmaceutical Sciences (J.-i.S., H.I., K.M., A.A.) and Laboratory of Organic \\ Chemistry, School of Pharmaceutical Sciences (M.S., Y.S.), University of Shizuoka, Shizuoka, Japan
}

Received March 6, 2019; accepted August 20, 2019

\begin{abstract}
In the research field of tubulin-binding agents for the development of anticancer agents, hidden targets are emerging as a problem in understanding the exact mechanisms of actions. The quinazoline derivative 1-(4-methoxyphenyl)-1-(quinazolin-4yl)ethan-1-ol (PVHD121) has anti-cell proliferative activity and inhibits tubulin polymerization by binding to the colchicine site of tubulin. However, the molecular mechanism of action of PVHD121 in cells remains unclear. Here, we demonstrate that PVHD121 delays mitotic entry and efficiently causes mitotic arrest with spindle checkpoint activation, leading to subsequent cell death. The dominant phenotype induced by PVHD121 was aberrant spindles with robust microtubules and unseparated centrosomes. The microtubules were radially distributed, and their ends appeared to adhere to kinetochores, and not to centrosomes. Extensive inhibition by high concentrations of PVHD121 eliminated all microtubules from cells. PVHD277 [1(4-methoxyphenyl)-1-(2-morpholinoquinazolin-4-yl)ethan-1-ol], a PVHD121 derivative with fluorescence, tended to localize close to the centrosomes when cells prepared to enter mitosis. Our results show that PVHD121 is an antimitotic agent that selectively disturbs microtubule formation at centrosomes during mitosis. This antimitotic activity can be attributed to the targeting
\end{abstract}

of centrosome maturation in addition to the interference with microtubule dynamics. Due to its unique bioactivity, PVHD121 is a potential tool for studying the molecular biology of mitosis and a potential lead compound for the development of anticancer agents.

\section{SIGNIFICANCE STATEMENT}

Many tubulin-binding agents have been developed as potential anticancer agents. The aim of this study was to understand the subcellular molecular actions of a quinazoline derivative tubulinbinding agent, 1-(4-methoxyphenyl)-1-(quinazolin-4-yl)ethan-1ol (PVHD121). As expected from its binding activity to tubulin, PVHD121 caused aberrant spindles and inhibited mitotic progression. However, in addition to tubulin, PVHD121 also targeted an unexpected biomolecule involved in centrosome maturation. Due to targeting the biomolecule just before entering mitosis, PVHD121 preferentially inhibited centrosome-derived microtubules rather than chromosome-derived microtubules during spindle formation. This study not only revealed the molecular action of PVHD121 in cells but also emphasized the importance of considering possible tubulin-independent effects of tubulinbinding agents via hidden targeted biomolecules for future use.

\section{Introduction}

Understanding the mechanism of action of small molecules in cells is crucial for their development as molecular probes for pharmacological research and as drug candidates in preclinical and clinical study. Targeted inhibitors often have offtarget biomolecules in addition to the intended target, which sometimes affects cells via both the intended and unintended

This study was supported by a "Drug Discovery Program" grant from Shizuoka Pharma Valley Center, and the Japan Society for the Promotion of Science KAKENHI [Grants 18710188 and 24590140].

The authors declare that there is no conflict of interest regarding the publication of this article.

${ }^{3}$ Current affiliation: Department of Chemistry and Life Science, School of Advanced Engineering, Kogakuin University, Japan.

${ }^{4}$ Current affiliation: Department of Materials and Life Sciences, Faculty of Science and Technology, Sophia University, Japan.

https://doi.org/10.1124/mol.119.116624.

S This article has supplemental material available at molpharm. aspetjournals.org. targets at the same concentration range. The importance of clarifying the exact mechanisms underlying the desired and unexpected activities of the inhibitors is increasingly recognized (Blagg and Workman, 2014, 2017).

In cancer research, various tubulin-binding agents (TBAs) have been developed with the aim to kill malignant cells (Dumontet and Jordan, 2010). Microtubules are dynamic cytoskeletal components assembled from $\alpha$ - and $\beta$-tubulin heterodimers. Together with their associated proteins, they are involved in many cellular processes, such as intracellular transport, cell shape maintenance, and cell division (Desai and Mitchison, 1997; Nogales, 2001). TBAs inhibit the assembly and disassembly of microtubules (Dumontet and Jordan, 2010; Amos, 2011; Stanton et al., 2011). Among these agents, vinca alkaloids and taxanes have been widely applied for the treatment of several types of cancers, and colchicine and nocodazole have been used as molecular tools for analyzing the molecular biology of cells. Recent structural biology has

ABBREVIATIONS: CCD, charge-coupled device; DAPI, XXX; HRP, horseradish peroxidase; PBS, phosphate-buffered saline; PVHD121, 1-(4methoxyphenyl)-1-(quinazolin-4-yl)ethan-1-ol; PVHD277, 1-(4-methoxyphenyl)-1-(2-morpholinoquinazolin-4-yl)ethan-1-ol; TBA, tubulin-binding agent. 
revealed six TBA-binding sites on tubulin, including the taxane site, the vinca site, and the colchicine site. It also indicates that TBAs targeting the different sites of tubulin have different molecular actions on microtubule assembly/ disassembly (Steinmetz and Prota, 2018). Molecular biological study has demonstrated that at low and clinically relevant concentrations, TBAs affect microtubule dynamics, rather than the cellular microtubule mass, regardless of their microtubule-stabilizing or -destabilizing activity at high concentrations (Dumontet and Jordan, 2010; Amos, 2011; Stanton et al., 2011). Inhibition of microtubule dynamics by TBAs slows down the mitotic progression of dividing cells or impairs proper spindle formation to cause aberrant mitosis with spindle checkpoint activation, consequently resulting in long-lasting mitosis and subsequent apoptotic cell death (Gadde and Heald, 2004; Clarke and Allan, 2009). This is the strategy in chemotherapies to combat malignant cells (Dumontet and Jordan, 2010; Steinmetz and Prota, 2018).

Mitotic phenotypes induced by individual TBAs exhibit slight differences, even if TBAs show the same effect on tubulin polymerization (Dumontet and Jordan, 2010; Stanton et al., 2011). This is apparently related to recent findings that small-molecule protein kinase inhibitors unintentionally bind to tubulin and inhibit microtubule dynamics (Munoz, 2017; Tanabe, 2017). For example, tivantinib was developed as an inhibitor against a receptor tyrosine kinase c-Met (Munshi et al., 2010). However, follow-up detailed studies have shown that the cytotoxic activity is independent of the kinase inhibition and results from the direct binding to tubulin and inhibiting microtubule dynamics (Basilico et al., 2013; Katayama et al., 2013). The number of protein kinase inhibitors directly binding to tubulin is increasing. This, in turn, highlights the importance of conducting pharmacological research on TBAs and especially taking into account the existence of hidden targets. In the research field of TBAs, whether a TBA of interest has an inhibitory activity against the cell cycle is examined using cultured cells. Once antimitotic activity of the given TBA is confirmed, further detailed investigation on the cellular effects, such as phenotype analyses, is often neglected. Thus, unexpected bioactivity resulting from the TBA binding to unintended biomolecules would be overlooked. To understand the exact action of individual TBAs in cells, detailed comparative approaches using TBAs binding to the same site on tubulin are needed (Munoz, 2017). A precise understanding of individual TBAs based on their effects on cells will lead to their efficient development as promising candidates of anticancer agents.

We recently reported that the quinazoline derivative 1-(4methoxyphenyl)-1-(quinazolin-4-yl)ethan-1-ol (PVHD121) (Fig. 1A) and its fluorescent derivative 1-(4-methoxyphenyl)-1-(2morpholinoquinazolin-4-yl)ethan-1-ol (PVHD277) (Fig. 6A) had submicromolar anti-cell proliferative $\mathrm{EC}_{50}$ values in various tumor-derived cell lines (Suzuki et al., 1998, 2017; Kuroiwa et al., 2015). We also showed that they directly bound to the colchicine site of tubulin and inhibited tubulin polymerization in vitro (Kuroiwa et al., 2015). The effects of PVHD121 on molecular events in cells remain largely unstudied, although tubulin is a plausible cellular target. For the future use of PVHD121 in the development of anticancer agents and molecular probes, it is important to elucidate the exact molecular mechanism of action of PVHD121 in cells. In this study, we focused on the effects of PVHD121 on the cell cycle, especially on mitotic progression. By detailed investigation of the PVHD121-induced phenotype and the subcellular distribution of PVHD277, we demonstrated the selective inhibitory activity of PVHD121 on microtubule formation at centrosomes during mitosis.

\section{Materials and Methods}

Chemicals and Antibodies. PVHD121 and PVHD277 were prepared as previously described (Suzuki et al., 1998, 2017; Kuroiwa et al., 2015). The purity of the compounds was confirmed on the basis of NMR spectra and melting points ( $>95 \%)$. Thymidine (PubChem ID: 5789), colchicine (PubChem ID: 6167), nocodazole (PubChem ID: 4122), and podophyllotoxin (PubChem ID: 10607) were purchased from Sigma-Aldrich (St. Louis, MO). All chemicals except thymidine were dissolved in DMSO and stored at $-20^{\circ} \mathrm{C}$ until use. Thymidine was dissolved in phosphate-buffered saline (PBS) and stored at $-20^{\circ}$ C. The primary antibodies were mouse monoclonal anti- $\alpha$-tubulin DM1A (catalog number T6199; Sigma-Aldrich), rabbit polyclonal antiphospho-histone H3 (Ser10, catalog number 06-570; Upstate, Temecula, CA), human antikinetochore CREST (catalog number 4200; MBL, Nagoya, Japan), mouse monoclonal anti- $\gamma$-tubulin 2GTU-88 (catalog number T5326; Sigma-Aldrich), rabbit polyclonal
A

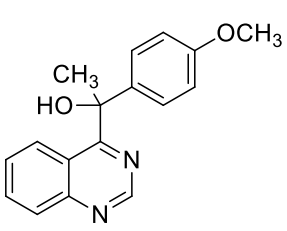

B
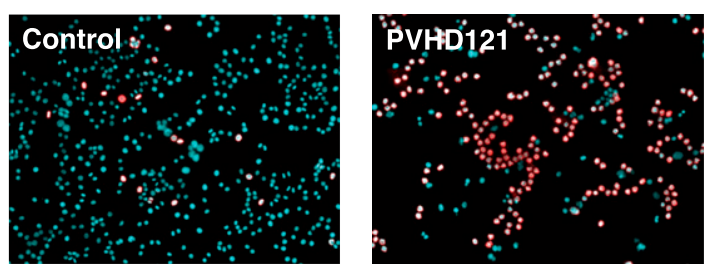

C

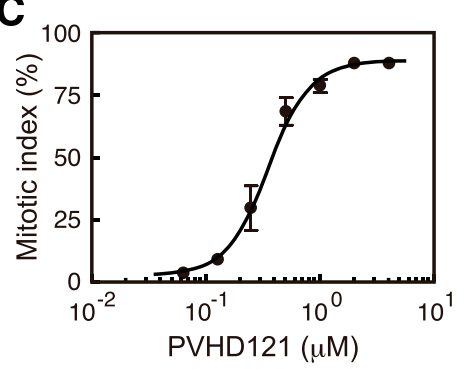

D

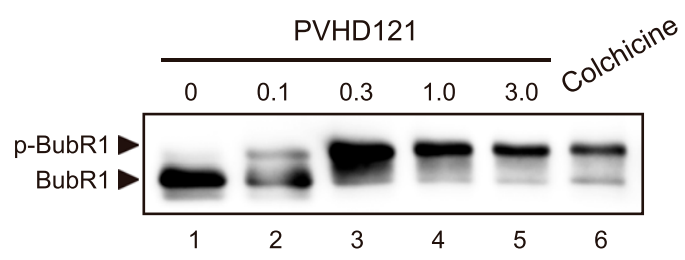

Fig. 1. PVHD121 is a potent mitotic inhibitor. (A) Chemical structure of PVHD121. (B) PVHD121 arrested HeLa cells at mitosis. Cells were treated with $4 \mu \mathrm{M}$ PVHD121 for 18 hours before fixation and staining with DAPI (blue) and anti-phospho-histone H3 (S10) antibody (red). Scale bar, $200 \mu \mathrm{m}$. (C) PVHD121 concentrationresponse curve in the mitotic arrest of HeLa cells. Graph, mean from three independent experiments; error bars, S.D. More than 100 cells were counted at each point. (D) PVHD121 activated the spindle checkpoint. Phosphorylation status of BubR1 was examined in HeLa cells treated with various concentrations of PVHD121 using a western blot analysis. The concentration (micromolar) used for each sample is indicated above lanes $1-5$. Colchicine $(50 \mathrm{nM})$ was used as a control (lane 6). The original image is shown in Supplemental Fig. 1. 
anti-TPX2 (catalog number N500-179; Novus, Littleton, CO), and mouse monoclonal anti-BubR1 (catalog number 612503; BD Biosciences, Franklin Lakes, NJ). The secondary antibodies were Alexa Fluor-conjugated goat anti-rabbit, anti-mouse, and anti-human $\operatorname{IgG}(\mathrm{H}+\mathrm{L})$ (Molecular Probes, Eugene, OR) and horseradish peroxidase (HRP)-linked sheep anti-mouse $\mathrm{F}\left(\mathrm{ab}^{\prime}\right)_{2}$ fragment (GE Healthcare, Piscataway, $\mathrm{NJ}$ ).

Cell Cultures. HeLa S3 cells were purchased from the RIKEN Cell Bank (Tsukuba, Japan). Hereafter, in this paper and the Supplemental Material, HeLa S3 cells are described as HeLa cells. HeLa cells were grown in Dulbecco's modified Eagle's medium (Wako, Osaka, Japan) supplemented with $10 \%$ fetal calf serum (Nichirei, Tokyo, Japan), 1\% penicillin-streptomycin (Invitrogen, Waltham, MA), sodium pyruvate (Invitrogen), and nonessential amino acids (Invitrogen) in a humidified atmosphere containing $5 \% \mathrm{CO}_{2}$ at $37^{\circ} \mathrm{C}$. Cell cycle synchronization was conducted using the double thymidine block method (Bostock et al., 1971). HeLa cells expressing fluorescent histone H2B (Sawada et al., 2016) or Plk1 (see Supplemental Material) were also maintained in the same manner. Before starting this study, we confirmed no contamination of mycoplasma in the cells using a MycoAlert Mycoplasma Detection Kit (Lonza, Basel, Switzerland).

Western Blot Analysis. HeLa cells were treated with various concentrations of PVHD121 for 18 hours and washed with PBS, and the cellular proteins were extracted for 30 minutes on ice using a buffer (50 mM Tris-HCl, pH 7.5; $150 \mathrm{mM} \mathrm{NaCl;1 \%} \mathrm{NP40)}$ supplemented by a protease and phosphatase inhibitor cocktail (Roche, Penzberg, Germany). Then, the cell lysates were centrifuged for 15 minutes at $20,000 \times g$. The concentration of soluble proteins in each supernatant was measured using the Bradford method (Bio-Rad, Hercules, CA). Equal amounts of each sample (10 $\mu \mathrm{g}$ of proteins) were resolved by SDS-PAGE, electrotransferred onto an Immobilon-P membrane (Millipore, Temecula, CA), and treated overnight with an anti-BubR 1 antibody at $4^{\circ} \mathrm{C}$. The membrane was rinsed and incubated with the secondary HRP-linked antibody. Finally, the HRP signals on the membrane were revealed using SuperSignal WestPico Substrate (Thermo Fisher, Waltham, MA) and detected using LAS3000 (GE Healthcare).

Immunostaining and Fluorescence Microscopy. HeLa cells were seeded at $1 \times 10^{5}$ cells per well in $500 \mu \mathrm{l}$ of medium on Laboratory-Tek II eight-well CC2 glass chamber slides (Nalge Nunc, Rochester, NY). After a 6-hour incubation at $37^{\circ} \mathrm{C}$ in a $5 \% \mathrm{CO}_{2}$ incubator, the culture medium was replaced with $400 \mu \mathrm{l}$ of fresh medium containing PVHD121, and the cells were incubated at $37^{\circ} \mathrm{C}$ in $5 \% \mathrm{CO}_{2}$ for $16-18$ hours. The final concentration of DMSO (the solvent for inhibitors) in the medium was $0.4 \%(\mathrm{v} / \mathrm{v})$. Before processing for immunofluorescence staining, HeLa cells were washed once with PBS and fixed with PBS containing $3 \%$ paraformaldehyde and $2 \%$ sucrose for 5 minutes and then with methanol at $-20^{\circ} \mathrm{C}$ for 10 minutes. Microtubules, mitotic chromosomes, and kinetochores were stained with anti- $\alpha$-tubulin, anti-phospho-histone H3 (Ser10), and CREST antibodies, respectively. The positions of centrosomes in cells were visualized using an anti- $\gamma$-tubulin antibody. The chromosome-driven microtubules were stained with an anti-TPX2 antibody. Primary antibodies were stained with Alexa Fluor-conjugated secondary antibodies. Finally, the cells were mounted using Vectashield mounting medium with DAPI (Vector Laboratories, Burlingame, CA). Fluorescent images were acquired using a DP30BW charge-coupled device (CCD) camera coupled to an IX71 microscope (Olympus, Tokyo, Japan) and MetaView imaging software (Molecular Devices, Downingtown, PA) and were superimposed using Adobe Photoshop (Adobe, San Jose, CA).

Evaluation of Antimitotic Activity and Mitotic Phenotypes. PVHD121-treated HeLa cells were sorted on the basis of an antiphospho-histone H3 (Ser10) antibody staining as "mitotic," "interphase," or "other" by visual inspection, and the percentage of mitotic cells for each inhibitor concentration was calculated. The PVHD121arrested mitotic HeLa cells were sorted by visual inspection according to staining pattern, using an anti- $\alpha$-tubulin antibody and DAPI (PubChem ID: 2954) for abnormal spindles or an anti- $\gamma$-tubulin antibody for unseparated centrosomes. The population sizes were determined for each phenotype at each inhibitor concentration. At least 100 cells were examined for each condition, and the concentration-response curves, $\mathrm{EC}_{50}$ values, and $\mathrm{EC}_{70}$ values were calculated using Microsoft Excel according to the median-effect theory (Chou, 2006). Concentration-response curves were fitted with a Hill equation using GraphPad Prism (GraphPad Software, La Jolla, CA).

Time-Lapse Microscopy. Synchronous HeLa cells expressing fluorescent histone H2B (Sawada et al., 2016) were seeded at 1.25 $\times 10^{5}$ cells per well in $400 \mu \mathrm{l}$ of the medium on Laboratory-Tek II eight-well CC2 glass chamber slides (Nalge Nunc). After a 7-hour incubation at $37^{\circ} \mathrm{C}$ in a $5 \% \mathrm{CO}_{2}$ incubator, the culture medium was replaced with $400 \mu \mathrm{l}$ of fresh medium containing PVHD121 or PVHD277 along with $50 \mathrm{mM}$ HEPES ( $\mathrm{pH} 7.4$ ). For the time-lapse detection of fluorescence derived from the fluorescent histone H2B in living cells, the medium was supplemented with BackDrop Background Suppressor (Molecular Probes) prior to imaging. Time-lapse imaging of living cells was performed at 1-minute or 2-minute intervals using MetaView. Fluorescence images were obtained using a DP30BW CCD camera coupled to an IX71 microscope using LUCPLFLN $40 \times$ as an objective lens (Olympus). A U-MWIBA3 mirror unit (Olympus, Tokyo, Japan) was used to detect the fluorescence of the recombinant histone $\mathrm{H} 2 \mathrm{~B}$.

Live Cell Imaging. Synchronous HeLa cells expressing fluorescent Plk1 (see Supplemental Material) on LAB-TEK II CC2 chamber glass slides (Nalge Nunc) were treated with $2 \mu \mathrm{M}$ PVHD277 and $0.8 \mu \mathrm{M}$ podophyllotoxin at least 20 minutes before fluorescence microscopic observation. In the competition assay, PVHD121 was additionally used at $20 \mu \mathrm{M}$. The medium was supplemented with $50 \mathrm{mM}$ HEPES (pH 7.4) and BackDrop Background Suppressor (Molecular Probes). Fluorescence images were obtained using a DP30BW CCD camera coupled to an IX71 microscope (Olympus) as previously described (Sawada et al., 2016; Suzuki et al., 2017). The images were colored and superimposed using Adobe Photoshop.

Statistical Analysis. The experiments under the same condition were designed to be repeated up to three times. All experiments were performed three times to allow statistical analysis, unless described in the figure legends. All data obtained from the experiments were subjected to statistical analysis. Each value was expressed as the mean \pm S.D., and statistical difference was considered significant when $P<0.05$.

\section{Results}

PVHD121 Causes Efficient Cell Cycle Arrest in Mitosis. Our previous flow cytometry analyses showed that PVHD121 caused the accumulation of G2/M phase cells (Kuroiwa et al., 2015). To examine whether PVHD121 arrests proliferating cells in M phase, HeLa cells were treated with this compound for 18 hours, corresponding to approximately one cell cycle. They were then immunostained with an antibody directed against phosphorylated histone H3 at Ser10, a mitotic cell marker (Hendzel et al., 1997). PVHD121 at $4 \mu \mathrm{M}$ caused a marked accumulation of mitotic cells to nearly the same extent as $100 \mathrm{nM}$ paclitaxel, the concentration causing the maximum mitotic arrest (Fig. 1B). PVHD121 showed clear concentration-dependent antimitotic activity; the $\mathrm{EC}_{50}$ for mitotic arrest was $0.45 \mu \mathrm{M}$ (Fig. 1C). An increase in the population of mitotic cells was observed at concentrations from 0.1 to $2.0 \mu \mathrm{M}$, whereas at higher concentrations, $>85 \%$ of the cells were arrested in mitosis. The spindle checkpoint was clearly activated at $1 \mu \mathrm{M}$ PVHD121, as judged by the phosphorylation status of the checkpoint protein 
BubR1 (Fig. 1D; Supplemental Fig. 1) (Elowe et al., 2007; Huang et al., 2008). DNA damage, however, was not observed in PVHD121-treated cells (Supplemental Fig. 2). Considering our previous result that the $\mathrm{EC}_{50}$ of PVHD121 for the inhibition of HeLa cell proliferation is $0.12 \mu \mathrm{M}$ (Kuroiwa et al., 2015), these results indicate that PVHD121 is a potent mitotic inhibitor in HeLa cells. The concentration-dependent antimitotic activity of PVHD121 was also observed in HCT116 cells; the $\mathrm{EC}_{50}$ for mitotic arrest was $1.5 \mu \mathrm{M}$ (Supplemental Fig. 3).

PVHD121 Delays Mitotic Entry and Causes Long-Lasting Mitotic Arrest. To investigate the effect of PVHD121 on the progression of mitosis, we performed timelapse microscopic observations using HeLa cells expressing recombinant histone $\mathrm{H} 2 \mathrm{~B}$ fused to a fluorescent protein (Sawada et al., 2016). The cells were synchronized in the cell cycle by double thymidine block (Bostock et al., 1971) and were exposed to PVHD121 at 7 hours after the release from the last thymidine block, when a cell cycle wave of the cells entered G2 phase. Chromosome morphology was detected by the fluorescent histone and was used to monitor the fate of individual cells. PVHD121-treated cells delayed mitotic entry by about 2 hours compared with the control cells (Fig. 2; Supplemental Fig. 4). After entering mitosis, they were arrested in mitosis for about 24.6 hours and subsequently died directly from aberrant mitosis (Fig. 2; Supplemental Fig. 4). Under our experimental conditions, we observed few cells that re-entered interphase without proper cell division. Colchicine and nocodazole, the well known TBAs binding to the colchicine site of tubulin, also retarded mitotic entry and caused aberrant mitosis for a long time before subsequent cell death (Fig. 2). These results show that PVHD121 prevents proper mitotic progression, similar to conventional TBAs (Dumontet and Jordan, 2010; Amos, 2011; Stanton et al., 2011). However, they also indicate that PVHD121-treated cells spend significantly much longer time in aberrant mitosis before cell death compared with the colchicine- or nocodazole-treated cells.

PVHD121 Induces Aberrant Spindles with Robust Microtubules in a Broad Concentration Range. To examine the effects of PVHD121 on spindle formation, the phenotypes induced by PVHD121 were observed and classified according to the morphology of spindle microtubules. Immunofluorescence staining was performed using an anti$\alpha$-tubulin antibody and DAPI (Fig. 3A). Treatment of HeLa cells with PVHD121 at concentrations below $2.0 \mu \mathrm{M}$, where there was a concentration-dependent increase in antimitotic activity, caused heterogeneous mitotic phenotypes, ranging from cells with bipolar spindles and misaligned chromosomes near spindle poles (bipolar-type; blue in Fig. 3B; also see Supplemental Fig. 5) to cells with single or multiple radial distributions of microtubules and misaligned chromosomes (radially arranged-type; red in Fig. 3B). At concentrations above $8 \mu \mathrm{M}$, two predominant mitotic phenotypes were observed: cells with multiple discrete puncta of $\alpha$-tubulin (puncta-type; green in Fig. 3B) and cells with diffuse $\alpha$-tubulin throughout the whole cell (diffuse-type; purple in Fig. 3B). The diffuse-type phenotype became the most prevalent population when HeLa cells were exposed to PVHD121 at concentrations above $16 \mu \mathrm{M}$, which was consistent with the conventional notion that TBAs binding to the colchicine sites of tubulin have a microtubule-destabilizing effect in cells (Dumontet and Jordan, 2010; Amos, 2011; Stanton et al., 2011). Cells with
A

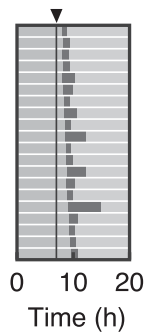

$4 \mu \mathrm{M}$ PVHD121
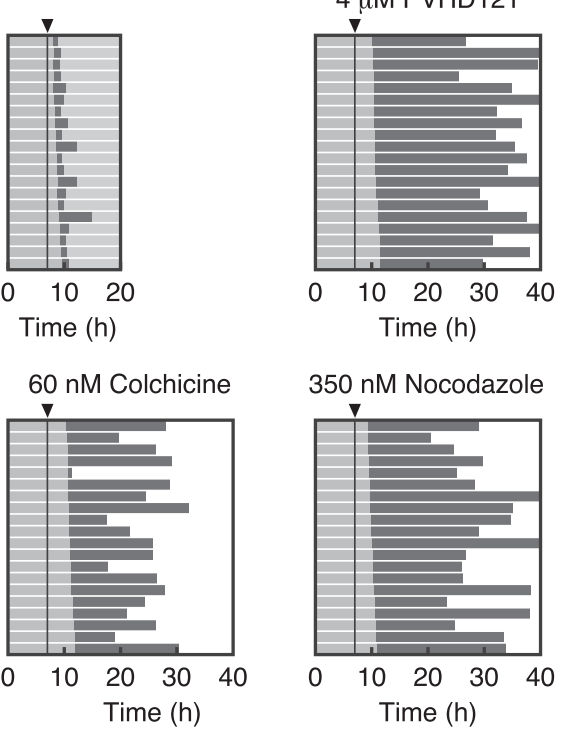

B

Timing of mitotic entry after thymidine release

\begin{tabular}{|c|c|c|c|}
\hline untreated & PVHD121 & Colchicine & Nocodazole \\
\hline $8 \mathrm{~h} 44 \mathrm{~min}$ & 10 h $42 \mathrm{~min}$ & $11 \mathrm{~h} 9 \mathrm{~min}$ & $10 \mathrm{~h} 10 \mathrm{~min}$ \\
\hline
\end{tabular}

C Duration of mitosis

\begin{tabular}{llll}
\hline untreated & PVHD121 & Colchicine & Nocodazole \\
\hline $1 \mathrm{~h} 5 \mathrm{~min}$ & $24 \mathrm{~h} 37 \mathrm{~min}$ & $14 \mathrm{~h} 36 \mathrm{~min}$ & $19 \mathrm{~h} \quad 8 \mathrm{~min}$
\end{tabular}

Fig. 2. Inhibition of mitosis progression by PVHD121. (A) Summary of the time-lapse observation of HeLa cells treated with $4 \mu \mathrm{M}$ PVHD121, $60 \mathrm{nM}$ colchicine, and $350 \mathrm{nM}$ nocodazole. The fate profiles of 20 representative cells treated with each inhibitor are presented. Each bar indicates an individual cell fate. The time after the last thymidine release is shown. Triangles indicate the time point ( 7 hours after thymidine release) for the addition of PVHD121 into the medium. Gray, dark gray, and white indicate interphase, mitosis, and cell death, respectively. (B) Median time point of the mitotic entry of cells treated with each mitotic inhibitor after the thymidine release. (C) Mitotic arrest duration of the cells treated with each mitotic inhibitor. Each median time of the cells from the beginning of the chromosome condensation until cell death is presented. PVHD121 showed statistically significant differences from untreated, colchicine, and nocodazole in mitotic entry (B) and mitosis duration $(\mathrm{C})(P<0.05$, Student's $t$ test). Detailed information on the results presented in Fig. 2 are shown in Supplemental Fig. 4.

multiple nuclei were rarely observed within the tested concentration range.

The population profile of the PVHD121-induced phenotypes in individual cells was compared with that of the colchicineinduced phenotypes (Fig. 3C). Colchicine began to increase the mitotic population at $10 \mathrm{nM}$, and the maximal mitotic population was reached at $50 \mathrm{nM}$ (Fig. 4A), which was consistent with previous reports (Bhattacharyya et al., 2008). The colchicine-induced spindle defects could also be assigned to one of four phenotypic categories, similar to the PVHD121-induced spindle defects shown in Fig. 3B. These two TBAs, however, exhibited a different concentrationdependent population profile of phenotypes at concentrations lower than those exerting the maximum population of mitotic cells ( $<2 \mu \mathrm{M}$ for PVHD121; $<50 \mathrm{nM}$ for colchicine). PVHD121 caused spindle defects, despite the presence of robust microtubules (bipolar and radially arranged phenotypes; Fig. 3C). 
A
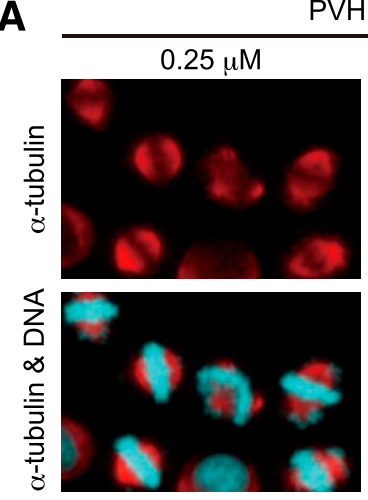

B
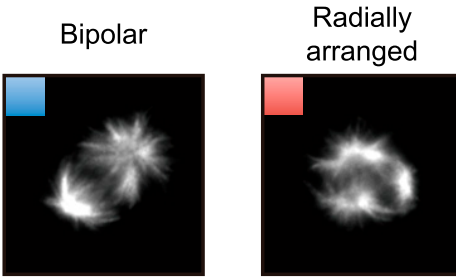

C

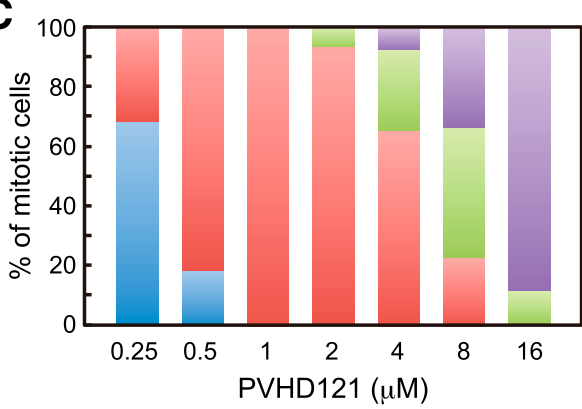

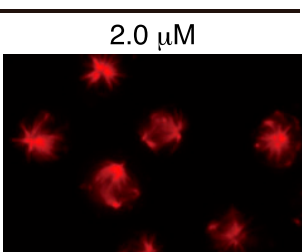

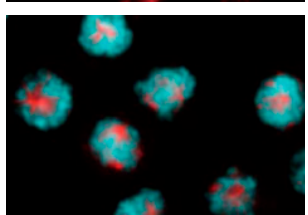

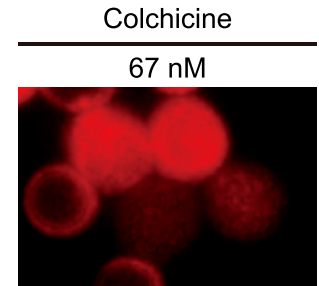

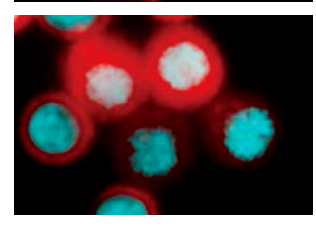

Diffuse
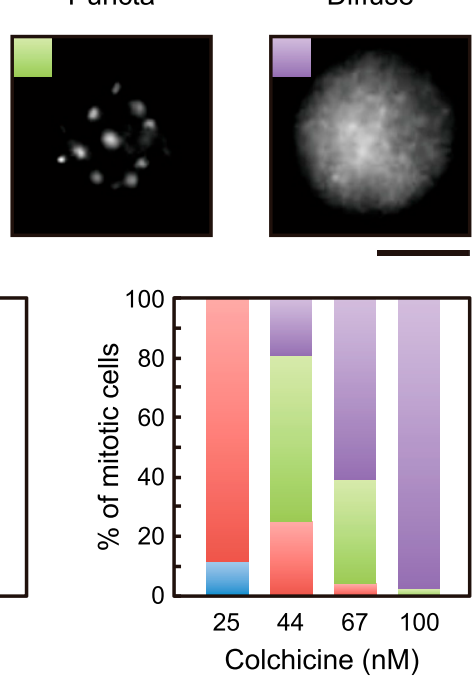

Fig. 3. Phenotypes of PVHD121-arrested mitotic cells. (A) PVHD121-arrested mitotic cells had aberrant spindles with microtubules. The mitotic phenotypes induced by PVHD121 at the indicated concentrations were identified by staining with an anti- $\alpha$-tubulin antibody (red) and DAPI (cyan). Colchicine $(67 \mathrm{nM})$ was used for the comparison with PVHD121. Scale bar, $20 \mu \mathrm{m}$. (B) The four major PVHD121-induced phenotypes. The phenotypes were grouped into four categories based on the staining profiles obtained using an anti- $\alpha$-tubulin antibody and DAPI (see text; for more examples, see Supplemental Fig. 5). Each phenotype was named according to the distribution status of microtubules. Cells were stained with an anti- $\alpha$-tubulin antibody only. The "radially arranged" phenotype (red) has three radial distributions of microtubules. Scale bar, $10 \mu \mathrm{m}$. (C) Concentration-dependent phenotype of PVHD121treated cells. HeLa cells were treated with PVHD121 at concentrations ranging from 0.25 to $16 \mu \mathrm{M}$. As a control, colchicine was used in the concentration range of 25-100 $\mathrm{nM}$. The colors correspond to the phenotypes shown in (B). Note that $2 \mu \mathrm{M}$ PVHD121 and $67 \mathrm{nM}$ colchicine achieved the maximal level of mitotic arrest. Two independent experiments were performed, counting more than 40 cells in each condition.
On the other hand, the colchicine-induced phenotypes were predominantly aberrant spindles with no observable microtubules (puncta and diffuse phenotypes; Fig. 3C). Even at concentrations that were higher than that necessary for maximal mitosis-arresting activity, a portion of the PVHD121treated cells still had observable microtubule bundles. These results demonstrate that, in HeLa cells, PVHD121 causes mitotic arrest but still has a broad concentration range that allows microtubules to be extended and organized.

PVHD121 Antimitotic Action Is More Potent Than the Action Eliminating Microtubules from Cells. The concentration-dependent effects of PVHD121 on microtubule mass and on mitotic progression were compared. The PVHD121 concentration-response curves are shown in Fig. 4A (compare the red and the black lines), and the $\mathrm{EC}_{50}$ values are summarized in Fig. 4B. PVHD121 caused a concentrationdependent increase in the population of mitotic cells without detectable robust microtubules at $2 \mu \mathrm{M}$ and above. The $\mathrm{EC}_{70}$ was $9.9 \mu \mathrm{M}, 13$ times higher than the $\mathrm{EC}_{70}$ value for mitotic arrest (Fig. 4A). In contrast, colchicine $\mathrm{EC}_{70}$ values for microtubule disappearance and mitotic arrest were similar, 74 and $42 \mathrm{nM}$, respectively. Although PVHD121 and colchicine bound to the same site of tubulin (Suzuki et al., 2017), PVHD121 was different from colchicine in that the elimination of microtubules was much weaker than mitotic arrest activity. The microtubule-destabilizing activity of PVHD121 in cells was equivalent to the in vitro inhibitory activity against tubulin polymerization in terms of concentration (Kuroiwa et al., 2015). The antimitotic activity of PVHD121 may be attributable to unknown mechanisms beyond the tubulin-targeting mechanism underlying the antimitotic activity of TBAs, such as colchicine.

PVHD121 Selectively Inhibits Centrosome-Driven Microtubule Nucleation. Based on the previously described results, we hypothesized that PVHD121 would selectively inhibit the microtubules originating from particular microtubule nucleation sites. The proper and prompt assembly of bipolar spindles requires the cooperative organization of both centrosome- and chromosome-driven microtubules (O'Connell and Khodjakov, 2007; Meunier and Vernos, 2012). In contrast, the separation of centrosomes during early mitosis depends only on the microtubules emanating from centrosomes (Gadde and Heald, 2004; Kapoor, 2017). To examine the possibility that PVHD121 would selectively disturb centrosomedriven microtubules, we observed the separation status of centrosomes in individual mitotic cells arrested by PVHD121. An antibody against $\gamma$-tubulin was used to visualize the positions of centrosomes (Khodjakov and Rieder, 1999). PVHD121 induced a clear concentration-dependent decrease in the population of mitotic cells containing separated centrosomes in the concentration range from 0.5 to $2.0 \mu \mathrm{M}$ (Fig. 4A; Supplemental Fig. 6). At higher concentrations $(>2.0 \mu \mathrm{M})$, more than $85 \%$ of the cells contained unseparated centrosomes. The PVHD121 concentration for the maximum 
A


B

\begin{tabular}{cccc} 
& Mitotic & Centrosome & Microtubule \\
progression & separation & assembly \\
\hline PVHD121 $(\mu \mathrm{M})$ & 0.45 & 1.1 & 6.6 \\
Colchicine $(\mathrm{nM})$ & 34 & 48 & 69 \\
\hline
\end{tabular}
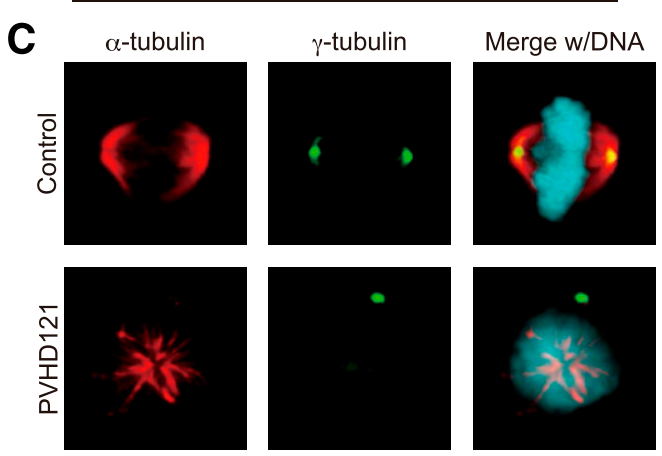

\section{D}
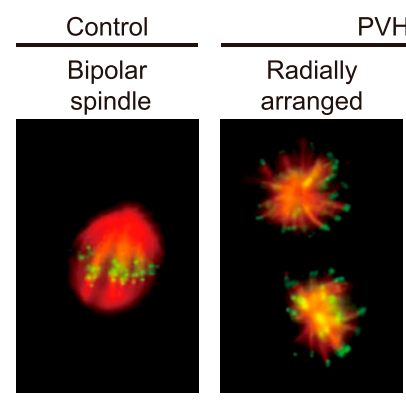

E
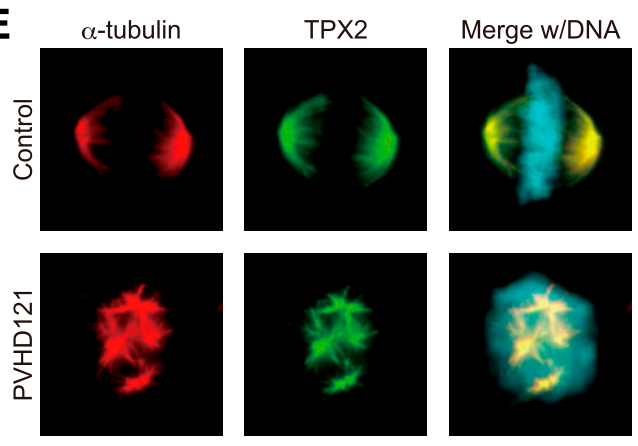

Fig. 4. PVHD121 selectively inhibits the centrosome-driven microtubules. (A) Concentration-response curves of PVHD121 (top) and colchicine (bottom) in the populations of mitotic cells (black, the same as Fig. 1C for PVHD121), mitotic cells with unseparated centrosomes (blue), and mitotic cells without microtubules (red). Graph, mean of two independent population of cells containing unseparated centrosomes was nearly equivalent to that for the maximum mitotic index, as shown in Fig. 4A (blue vs. black lines). The PVHD121-induced unseparated centrosomes were located near the periphery of individual cells and did not appear to coincide with microtubules (Fig. 4C). This result indicates that the antimitotic activity of PVHD121 results primarily from the inhibition of centrosome-driven microtubule formation.

The radially organized microtubules located at the site coincided with the spherical mass of chromosomes in the PVHD121-arrested mitotic cells (Fig. 4C). Next, we investigated the spatial relationship between microtubules and kinetochores, the chromosomal sites important for attachment and stabilization of the spindle microtubules. HeLa cells were treated with PVHD121 for 16 hours before being fixed and immunostained with anti- $\alpha$-tubulin and antikinetochore CREST antibodies (Mori et al., 1980). One end of each microtubule bundle appeared to be attached to a kinetochore (Fig. 4D). This observation suggests that the microtubules in PVHD121-arrested mitotic cells emanate from the chromosomes. This was supported by an additional immunofluorescence analysis showing that the radially distributed microtubules were highly colocalized with TPX2 (Fig. 4E), a microtubule-binding protein important for the formation of chromosome-driven microtubules (Gruss et al., 2002; Tulu et al., 2006).

These results show that PVHD121 efficiently inhibits centrosome-driven microtubule formation at lower concentrations than those required to eliminate all microtubules from cells, suggesting that PVHD121-mediated selective inhibition in centrosome-driven microtubules is likely to be a significant factor in the antimitotic activity.

PVHD121 Slows Chromosome-Driven Microtubule Formation. The treatment of HeLa cells with high concentrations of PVHD121 (2-8 $\mu \mathrm{M})$ often induced discrete puncta of $\alpha$-tubulin in mitotic cells. The $\alpha$-tubulin structures were scattered over the area occupied by the chromosomes (Supplemental Fig. 5) and colocalized well with kinetochores (Fig. 4D). To determine whether the $\alpha$-tubulin structures would have the potential to form microtubules in the presence of PVHD121, HeLa cells were synchronized in the cell cycle by a thymidine double block and were exposed to $4 \mu \mathrm{M}$ PVHD121 after the second thymidine release. The cells were fixed at 8 , 10, or 14 hours after thymidine release (Fig. 5A) and immunostained with anti- $\alpha$-tubulin and CREST antibodies. At 8 hours after thymidine release, $82 \%$ of the mitotic cells had the $\alpha$-tubulin punctum structures surrounded by several

experiments. More than 40 cells were counted at each point. (B) $\mathrm{EC}_{50}$ values for PVHD121 and colchicine for the inhibition of mitotic progression, centrosome separation, and mitotic microtubule formation, based on the concentration-response curves shown in (A). (C) Representative radially arranged microtubules induced by $1 \mu \mathrm{M}$ PVHD121 (bottom) are shown with centrosomes; $\alpha$-tubulin is labeled in red, $\gamma$-tubulin in green, and DNA in cyan. Normal spindle microtubules at metaphase are also shown as a control (top). (D) Radially arranged microtubules and $\alpha$-tubulin puncta are shown with kinetochores: $\alpha$-tubulin is labeled in red and CREST signals in green. (E) Representative radially arranged microtubules in PVHD121-arrested mitosis (bottom) were highly colocalized with TPX2: $\alpha$-tubulin is labeled in red, TPX2 in green, and DNA in cyan. Normal spindle microtubules at metaphase are also shown as a control (top). Scale bar, $10 \mu \mathrm{m}$. 
A

\begin{tabular}{|c|c|c|}
\hline $\begin{array}{c}0 \mathrm{~h} \\
\mathrm{G} 1 / \mathrm{S}\end{array}$ & $\begin{array}{c}4 \mathrm{~h} \\
\mathrm{~S}\end{array}$ & $\begin{array}{c}8 \mathrm{~h} \\
\text { M-arrest }\end{array}$ \\
\hline & 个 & Treatment \\
\hline $\begin{array}{l}\text { Release } \\
\text { from } \\
\text { thymidine }\end{array}$ & & $\begin{array}{l}\downarrow \\
\text { Fixation }\end{array}$ \\
\hline
\end{tabular}

B
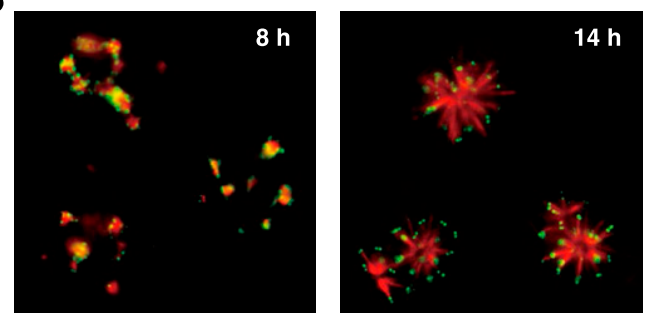

c
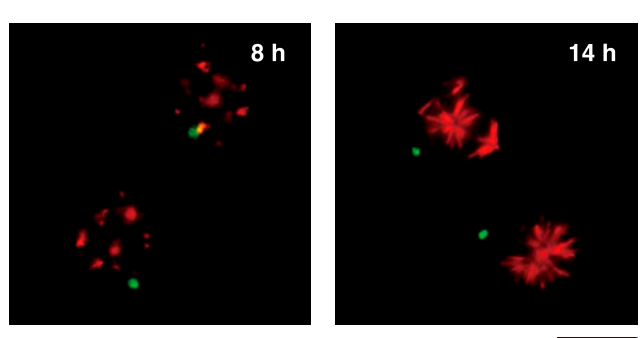

Fig. 5. PVHD121 slows down the formation of chromosome-driven microtubules. (A) Time course of the experimental procedure. (B and C) Immunostaining images of HeLa cells treated with $4 \mu \mathrm{M}$ PVHD121 at 8 and 14 hours after the last thymidine release. The anti- $\alpha$-tubulin antibody signal is shown in red, and CREST (B) and anti- $\gamma$-tubulin antibody (C) signals are shown in green. Scale bar, $10 \mu \mathrm{m}$.

kinetochores (Fig. 5B). The remaining mitotic cells had radially distributed and very short microtubules. At 10 hours after the thymidine release, the population of the cells with the kinetochore-associated $\alpha$-tubulin structures decreased to $78 \%$ of the mitotic cells. Under prolonged mitotic arrest (14 hours after thymidine release), almost all PVHD121-arrested mitotic cells had radially organized microtubules, the ends of which were associated with kinetochores (Fig. 5B). Few microtubules coincided with the centrosomes (Fig. 5C). More than half $(57 \%)$ of mitotic cells contained a single radial array of microtubules. These results indicate that the $\alpha$-tubulin puncta are not nonfunctional aggregates of $\alpha$-tubulin damaged by PVHD121 but have the potential to extend microtubules. Considering that proper mitosis is completed in about 1.2 hours (Fig. 2, A and C), these results show that extensive inhibition by PVHD121 at high concentrations slows down the formation and assembly of chromosome-driven microtubules.

PVHD277 Localizes around Centrosomes in the Early Stage of Mitosis. To investigate the possibility that PVHD121 would exert antimitotic activity by a mechanism other than the tubulin-targeting action, we examined the intracellular behavior of PVHD277, a PVHD121 derivative with greater fluorescence activity than PVHD121 (Fig. 6A; Supplemental Fig. 7), by live cell imaging. PVHD277 could act as a fluorescent probe to trace its distribution in cultured cells (Supplemental Fig. 7; Suzuki et al., 2017). PVHD277 was similar to PVHD121 with respect to biological activities, such as tubulin-binding, mitotic entry retardation, and mitotic arrest activities, including the mitotic phenotype (Supplemental Fig. 8; Suzuki et al., 2017). It was similar to PVHD121 in that it caused mitotic arrest at lower concentrations than those required for the elimination of microtubules from mitotic cells (Suzuki et al., 2017). Synchronous HeLa cells expressing a fluorescent protein-tagged Plk1 were exposed to PVHD277 with an excess amount of podophyllotoxin before a cell cycle wave of cells entered mitosis (at 7 hours after the last thymidine release). Fluorescent Plk1 worked as an indicator of the centrosome position from G2 phase to metaphase of mitosis (Supplemental Fig. 9; Kishi et al., 2009). Podophyllotoxin was a nonfluorescent TBA targeting the colchicine site of tubulin with higher affinity than PVHD277 (Suzuki et al., 2017) and was considered to be useful to easily observe the subcellular distribution of PVHD277 under conditions in which PVHD277 could not bind to tubulin. PVHD277 fluorescence was predominantly observed in the cytoplasm before the nuclear envelope was broken (Supplemental Fig. 7), but the distribution of PVHD277 in individual cells was more heterogeneous than expected (Fig. 6, B-D). Therefore, we classified the distribution of PVHD277 based on the centrosome position in individual cells, as shown in Fig. 6, B and C. First, we roughly classified the subcellular distribution of PVHD277 into two groups, one consisting of cells whose PVHD277 fluorescence was located around the centrosomes (Fig. 6B) and another consisting of cells whose PVHD277 fluorescence was not (Fig. 6C). The former group was further divided into two types of cells, one with a focus of PVHD277 fluorescence on or just adjacent to the centrosomes (blue in Fig. 6B), and another with relatively dense distribution of PVHD277 fluorescence surrounding the centrosomes (light blue in Fig. 6B). The latter group, whose PVHD277 was not located around the centrosomes, was further classified into three subgroups: cells with a diffuse distribution of PVHD277 fluorescence in whole cytoplasm (dark green in Fig. 6C), cells with multiple foci of PVHD277 fluorescence (green in Fig. 6C), and cells with a ring-shaped distribution of PVHD277 in cells (light green in Fig. 6, C and D). Based on fluorescence microscopic observation using the synchronized cells, the time-course population profile of the subcellular PVHD277 distribution pattern was determined, as summarized in Fig. 6E. In 8-9 hours after thymidine release, more than half of the cells (27 out of 48 cells) appeared to have a focus of PVHD277 fluorescence at the same position on or near the unseparated centrosomes. However, the population of such cells decreased over time. From 10 to 12 hours after thymidine release, a small portion of the cells began to condense chromosomes in the experimental conditions (Supplemental Fig. 10), and a large number of cells (20 out of 49 cells) had a ring-shaped pattern of PVHD277 fluorescence (Fig. 6E). Using HeLa cells expressing the fluorescent recombinant histone H2B (Sawada et al., 2016), the ring-shaped fluorescence of PVHD277 was detected around the spherical mass of condensed chromosomes (Fig. 6D). Twelve hours after the thymidine release, when some of the cells dispersed the nuclear envelope and struggled to form a proper spindle (Supplemental Fig. 10), we observed that a large number of cells had PVHD277 fluorescence throughout the whole cell. Some of those cells contained discrete puncta of PVHD277 fluorescence. We were unable to find evidence of the subcellular localization of PVHD277 puncta. The additional use of an excess amount of PVHD121 
A

B
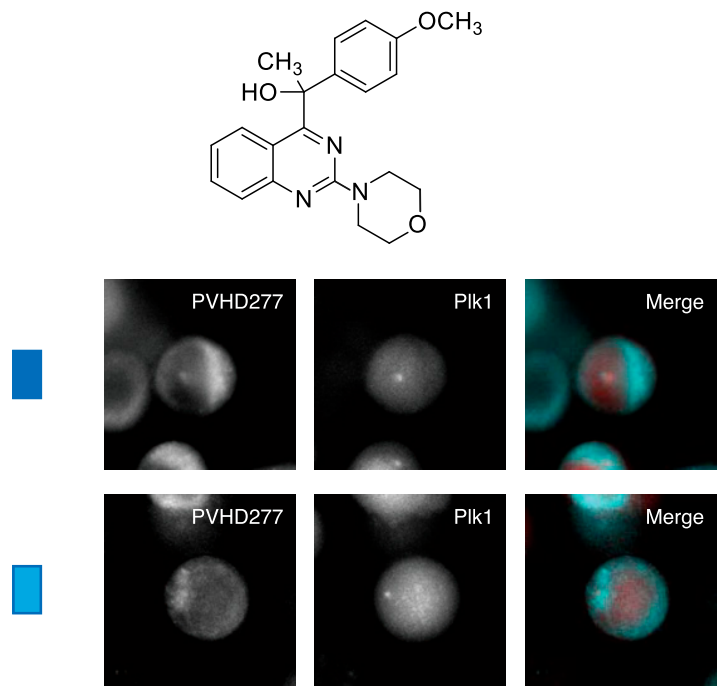

C
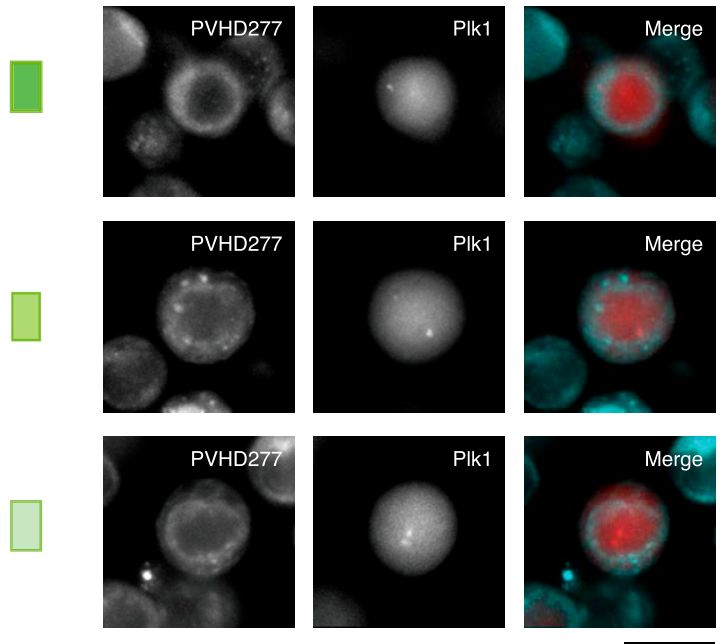

D
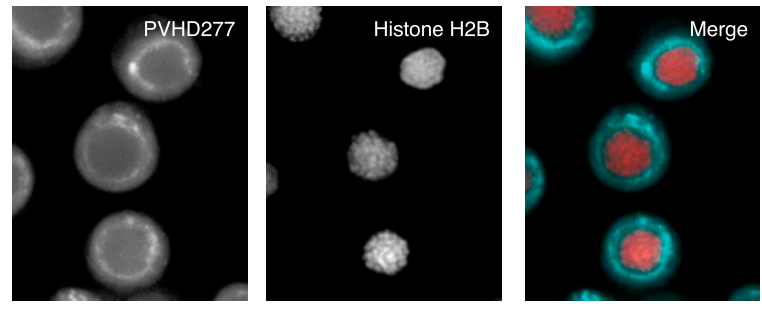

$\mathbf{E}$

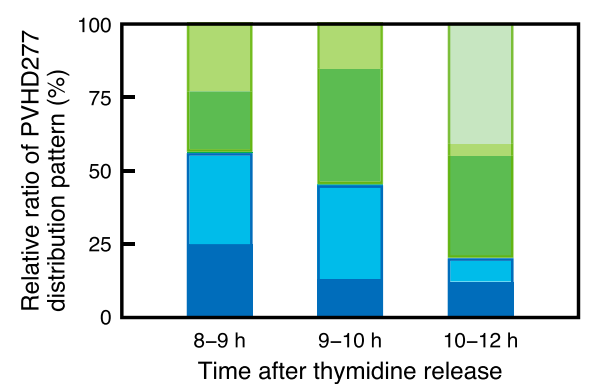

Fig. 6. Subcellular distribution of PVHD277 in HeLa cells. (A) Chemical structure of PVHD277. (B) Two major distribution patterns of PVHD277 fluorescence around the centrosomes. Representative fluorescence microscopy images of PVHD277-treated HeLa cells expressing fluorescent Plk1. The images were taken 8-12 hours after the thymidine release. (C) Three or no use of podophyllotoxin along with PVHD277 in the live imaging assay decreased the population of cells with PVHD277 fluorescence at the same position on or near the centrosomes and the population of cells with ring-shaped PVHD277 fluorescence (Supplemental Fig. 11). This confirmed the spatiotemporal localization of PVHD121 and PVHD277 in the early phase of mitosis. These results indicate that the two PVHD compounds tend to localize around the centrosomes and the nuclear membrane when cells prepare to enter mitosis, probably by targeting particular proteins other than tubulin.

\section{Discussion}

In this study, the quinazoline derivative PVHD121 was characterized as an antimitotic agent that operated in a nanomolar/liter to micromolar/liter range of concentrations. The most prominent biological characteristic of PVHD121 was the selective inhibition of centrosome-driven microtubule formation during spindle formation in mitosis, which was different from a conventional TBA colchicine. This unique activity can be attributed to the accumulation of PVHD121 around the centrosomes just before mitotic entry.

The results of this study indicate that PVHD121 antimitotic activity is primarily due to the inhibition of centrosome-derived microtubule formation in mitosis. The cell cycle-specific localization of PVHD121 around the centrosomes can help us understand the molecular action of PVHD121 (Fig. 6; Supplemental Fig. 11). That is to say, it is hypothesized that PVHD121 inhibits a molecular event involving the centrosomes just prior to mitotic entry, consequently causing failure of microtubule formation at the centrosomes during spindle formation. Centrosome maturation is a promising candidate for the molecular event targeted by PVHD121. This process is driven by the recruitment and activation of $\gamma$-tubulin and other proteins that form the pericentriolar material around the centrioles (Woodruff et al., 2014; Conduit et al., 2015). Some reports showed that the inhibition of pericentriolar material components caused a mitotic phenotype similar to the PVHD121-induced phenotype. RNA interference targeting Cep192 (centrosomal protein of $192 \mathrm{kDa}$ ) mRNA inhibited centrosome maturation, which led to aberrant spindles consisting of microtubules organized around chromosomes, but not from centrosomes (GomezFerreria et al., 2007). A small compound targeting TACC3 (transforming acidic coiled-coil protein 3), spindlactone, induced aberrant multipolar spindles by selectively inhibiting

major distribution patterns of PVHD277 fluorescence that is not located around the centrosomes. (D) Representative fluorescence microscopy images of PVHD277-treated HeLa cells expressing fluorescent histone H2B. The images were taken 10-12 hours after the thymidine release. Cyan and red in the merged images show the fluorescence derived from PVHD277 and fluorescent Plk1 (B and C) or histone H2B (D), respectively. Plk1-derived fluorescence was not clearly observed in some cells, probably due to low expression level. Note that the fluorescence of recombinant Plk1 at the centrosome position was not affected by PVHD121. Scale bar, $20 \mu \mathrm{m}$. (E) Time-course population profile of PVHD277-treated cells according to the PVHD277-derived fluorescence distribution pattern in each cell. The colors in the graph correspond to the cells shown in (B) and (C). PVHD277 was used at $2 \mu \mathrm{M}$ with $0.8 \mu \mathrm{M}$ podophyllotoxin. Two independent experiments were performed, counting more than 40 cells in each time point. 
the formation of centrosome-driven microtubules (Yao et al., 2014). These reports support the hypothesis that PVHD121 would target a protein involved in centrosome maturation and prevent centrosomes from increasing microtubule-organizing activity at low effective concentrations. The hypothesis is consistent with our previous results showing that PVHD121 does not appear to affect interphase microtubules (Kuroiwa et al., 2015). The results described here cannot eliminate the possibility that PVHD121 could affect mitotic molecular events other than centrosome maturation. When HCT116 cells were used instead of HeLa cells, the PVHD121-induced mitotic phenotype also changed from the bipolar type to the diffuse type in a concentration-dependent manner, as in the case of HeLa cells (Supplemental Fig. 3). At the concentrations lower than those exerting the maximum antimitotic activity $(<8 \mu \mathrm{M})$, the puncta-type cells were often observed in addition to the bipolar- and radially arranged-type cells. This may be due to the difference in the ability of chromosome-driven microtubules to form between HeLa and HCT116 cells. This result suggests that in various cultured cells, selective inhibition of centrosome-driven microtubule formation could be involved in the antimitotic activity of PVHD121. Further studies are required to elucidate the molecular mechanisms that render centrosome-driven microtubule organization more susceptible to PVHD121 in mitotic cells compared with chromosome-driven microtubule formation. The molecular mechanism by which PVHD121, as well as TBAs targeting the colchicine site, induces a delay in the start of chromosome condensation also remains unclear. This may be due to the orchestrated progression of the molecular events involved in mitosis. The specific localization of PVHD121 and PVHD277 around a spherical mass of condensed chromosomes may provide insight into how the compounds retard mitotic chromosome condensation.

This study provides an application of fluorescent inhibitors. Fluorescent bioactive compounds can be used to visualize subcellular distributions and can provide information about when and where they function in cells. We successfully identified the subcellular distribution of PVHD121 in mitosis using PVHD277 (Fig. 6). The key to success was the quinazoline core of PVHD121. A quinazoline core can function as a pharmacophore in medicinal chemistry and as a fluorophore in material chemistry (Achelle et al., 2014; Khan et al., 2016), thereby facilitating the development of the fluorescent derivative PVHD277 based on the structure-activity relationship study for PVHD121 (Suzuki et al., 2017). When preparing a fluorescent probe by adding a fluorophore to an inhibitor, the reduction or loss of inhibitory activity often becomes an issue. The strategy of developing an inhibitor using a fluorophore as a pharmacophore can avoid such a problem in the preparation of fluorescent probes. To facilitate the development of fluorescent inhibitors against various proteins, it is necessary to develop various types of fluorescent chromophores that can be used as pharmacophores.

The mitotic phenotype induced by PVHD121 reinforces the idea that small chemical compounds can be used to dissect complex molecular events, such as spindle assembly during mitosis, and can be valuable tools for molecular biology research (Peterson and Mitchison, 2002). Recent studies of spindle microtubules in higher eukaryotic cells have indicated that spindle microtubules nucleate from three distinct sites: centrosomes, chromosomes, and microtubules (O'Connell and
Khodjakov, 2007; Meunier and Vernos, 2012). They cooperatively organize themselves into a spindle along with microtubule-associated proteins. Using PVHD121, it is possible to easily prepare mitotic cells lacking centrosome-driven microtubules. Thereby, PVHD121 can be a useful tool for studying the molecular biology of the mitotic spindle, such as analyses of microtubules derived from chromosomes. Additionally, it can be a tool for screening for novel small compounds that selectively inhibit chromosome-derived microtubule formation.

Due to the discovery of taxanes and vinca alkaloids, tubulin has been recognized as a good target protein for the development of anticancer agents (Dumontet and Jordan, 2010). They bind to another site of tubulin, not to the colchicine site. To date, TBAs that target the colchicine site have not been approved as anticancer agents, although colchicine-site TBAs are probably the most extensively studied among the six binding-site classes of TBAs (Steinmetz and Prota, 2018). The colchicine site of tubulin is fairly deep, large in size, and predominantly hydrophobic in nature. It is pointed out that, due to this feature, the colchicine site tends to be a potential binding site for a wide range of small-molecule compounds. It is not surprising that colchicine-site TBAs have effects on cells through binding to nontubulin biomolecules. Therefore, in the development of anticancer agents based on colchicine-site TBAs, it is quite important and necessary to elucidate whether they affect nontubulin biomolecules. Once the existence of a tubulin-independent mechanism of action is found, the TBAs will be more properly evaluated as candidates of anticancer agents. Considering that PVHD121 targets centrosome maturation as suggested in this manuscript, PVHD121 is expected to be a good candidate for anticancer agents. Plk1 and Aurora kinase A, both of which play an important role in centrosome maturation, have been recognized as good targets for anticancer agents (Rivera-Rivera and Saavedra, 2016). However, the antimitotic activity of PVHD121 required higher concentrations compared with taxanes and vinca alkaloids, as well as with the other known colchicine-site TBAs, colchicine and nocodazole. Further efforts are needed to develop more potent compounds through the structure-activity relationship research based on PVHD121.

In conclusion, this study shows that PVHD121 is an antimitotic agent causing aberrant spindle formation and subsequent cell death. The antimitotic activity is primarily attributed to the targeting of the centrosome function to form microtubules, rather than the interference with microtubule dynamics by binding to tubulin. Small compounds that selectively inhibit particular functions of microtubules may get around the undesirable side effects associated with conventional anticancer TBAs. In this respect, the novel microtubule inhibitor PVHD121 can be regarded as a potential lead compound for the development of anticancer agents. Detailed analysis of the molecular action of TBAs will contribute to their future proper use and enable an identification of new drug target biomolecules for anticancer and antimitotic agents.

\footnotetext{
Authorship Contributions

Participated in research design: Sawada, Asai.

Conducted experiments: Sawada, Ishii.

Contributed new reagents or analytical tools: Matsuno, Sato, Suzuki.
} 
Performed data analysis: Sawada, Ishii, Asai.

Wrote or contributed to the writing of the manuscript: Sawada, Ishii, Asai.

\section{References}

Achelle S, Rodríguez-López J, and Robin-le Guen F (2014) Synthesis and photophysical studies of a series of quinazoline chromophores. J Org Chem $\mathbf{7 9}$ $7564-7571$

Amos LA (2011) What tubulin drugs tell us about microtubule structure and dynamics. Semin Cell Dev Biol 22:916-926.

Basilico C, Pennacchietti S, Vigna E, Chiriaco C, Arena S, Bardelli A, Valdembri D, Serini G, and Michieli P (2013) Tivantinib (ARQ197) displays cytotoxic activity that is independent of its ability to bind MET. Clin Cancer Res 19:2381-2392.

Bhattacharyya B, Panda D, Gupta S, and Banerjee M (2008) Anti-mitotic activity of colchicine and the structural basis for its interaction with tubulin. Med Res Rev 28 $155-183$.

Blagg J and Workman P (2014) Chemical biology approaches to target validation in cancer. Curr Opin Pharmacol 17:87-100.

Blagg J and Workman P (2017) Choose and use your chemical probe wisely to explore cancer biology. Cancer Cell 32:9-25.

Bostock CJ, Prescott DM, and Kirkpatrick JB (1971) An evaluation of the double thymidine block for synchronizing mammalian cells at the G1-S border. Exp Cell Res 68:163-168.

Chou TC (2006) Theoretical basis, experimental design, and computerized simulation of synergism and antagonism in drug combination studies. Pharmacol Rev $\mathbf{5 8}$ $621-681$.

Clarke PR and Allan LA (2009) Cell-cycle control in the face of damage--a matter of life or death. Trends Cell Biol 19:89-98.

Conduit PT, Wainman A, and Raff JW (2015) Centrosome function and assembly in animal cells. Nat Rev Mol Cell Biol 16:611-624.

Desai A and Mitchison TJ (1997) Microtubule polymerization dynamics. Annu Rev Cell Dev Biol 13:83-117.

Dumontet C and Jordan MA (2010) Microtubule-binding agents: a dynamic field of cancer therapeutics. Nat Rev Drug Discov 9:790-803.

Elowe S, Hümmer S, Uldschmid A, Li X, and Nigg EA (2007) Tension-sensitive Plk1 phosphorylation on BubR1 regulates the stability of kinetochore microtubule interactions. Genes Dev 21:2205-2219.

Gadde S and Heald R (2004) Mechanisms and molecules of the mitotic spindle. Curr Biol 14:R797-R805.

Gomez-Ferreria MA, Rath U, Buster DW, Chanda SK, Caldwell JS, Rines DR, and Sharp DJ (2007) Human Cep192 is required for mitotic centrosome and spindle assembly. Curr Biol 17:1960-1966.

Gruss OJ, Wittmann M, Yokoyama H, Pepperkok R, Kufer T, Silljé H, Karsenti E, Mattaj IW, and Vernos I (2002) Chromosome-induced microtubule assembly mediated by TPX2 is required for spindle formation in HeLa cells. Nat Cell Biol 4 871-879

Hendzel MJ, Wei Y, Mancini MA, Van Hooser A, Ranalli T, Brinkley BR, BazettJones DP, and Allis CD (1997) Mitosis-specific phosphorylation of histone H3 initiates primarily within pericentromeric heterochromatin during G2 and spreads in an ordered fashion coincident with mitotic chromosome condensation. Chromosoma 106:348-360.

Huang H, Hittle J, Zappacosta F, Annan RS, Hershko A, and Yen TJ (2008) Phosphorylation sites in BubR1 that regulate kinetochore attachment, tension, and mitotic exit. $J$ Cell Biol 183:667-680.

Kapoor TM (2017) Metaphase spindle assembly. Biology (Basel) 6

Katayama R, Aoyama A, Yamori T, Qi J, Oh-hara T, Song Y, Engelman JA and Fujita N (2013) Cytotoxic activity of tivantinib (ARQ 197) is not due solely to c-MET inhibition. Cancer Res 73:3087-3096.

Khan I, Zaib S, Batool S, Abbas N, Ashraf Z, Iqbal J, and Saeed A (2016) Quinazolines and quinazolinones as ubiquitous structural fragments in medicinal chemistry: an update on the development of synthetic methods and pharmacological diversification. Bioorg Med Chem 24:2361-2381.
Khodjakov A and Rieder CL (1999) The sudden recruitment of $\gamma$-tubulin to the centrosome at the onset of mitosis and its dynamic exchange throughout the cell cycle, do not require microtubules. J Cell Biol 146:585-596.

Kishi K, van Vugt MA, Okamoto K, Hayashi Y, and Yaffe MB (2009) Functional dynamics of Polo-like kinase 1 at the centrosome. Mol Cell Biol 29:3134-3150.

Kuroiwa K, Ishii H, Matsuno K, Asai A, and Suzuki Y (2015) Synthesis and structureactivity relationship study of 1-phenyl-1-(quinazolin-4-yl)ethanols as anticancer agents. ACS Med Chem Lett 6:287-291.

Meunier S and Vernos I (2012) Microtubule assembly during mitosis - from distinct origins to distinct functions? J Cell Sci 125:2805-2814.

Mori Y, Peebles C, Fritzler MJ, Steigerwald J, and Tan EM (1980) Autoantibody to centromere (kinetochore) in scleroderma sera. Proc Natl Acad Sci USA 77: $1627-1631$

Munoz L (2017) Non-kinase targets of protein kinase inhibitors. Nat Rev Drug Discov 16:424-440.

Munshi N, Jeay S, Li Y, Chen CR, France DS, Ashwell MA, Hill J, Moussa MM, Leggett DS, and Li CJ (2010) ARQ 197, a novel and selective inhibitor of the human c-Met receptor tyrosine kinase with antitumor activity. Mol Cancer Ther $\mathbf{9}$ : 1544-1553.

Nogales E (2001) Structural insight into microtubule function. Annu Rev Biophys Biomol Struct 30:397-420.

O'Connell CB and Khodjakov AL (2007) Cooperative mechanisms of mitotic spindle formation. J Cell Sci 120:1717-1722.

Peterson JR and Mitchison TJ (2002) Small molecules, big impact: a history of chemical inhibitors and the cytoskeleton. Chem Biol 9:1275-1285.

Rivera-Rivera Y and Saavedra HI (2016) Centrosome - a promising anti-cancer target. Biologics 10:167-176

Sawada JI, Osawa A, Takeuchi T, Kaneda M, Oishi S, Fujii N, Asai A, Tanino K, and Namba K (2016) Functional 1,3a,6a-triazapentalene scaffold: design of fluorescent probes for kinesin spindle protein (KSP). Bioorg Med Chem Lett 26 $5765-5769$.

Stanton RA, Gernert KM, Nettles JH, and Aneja R (2011) Drugs that target dynamic microtubules: a new molecular perspective. Med Res Rev 31:443-481.

Steinmetz MO and Prota AE (2018) Microtubule-targeting agents: strategies to hijack the cytoskeleton. Trends Cell Biol 28:776-792.

Suzuki Y, Sawada J, Hibner P, Ishii H, Matsuno K, Sato M, Witulski B, and Asai A (2017) Fluorescent anticancer quinazolines as molecular probes for $\beta$-tubulin colchicine site competition assay and visualization of microtubules as intracellular targeting sites. Dyes Pigm 145:233-238.

Suzuki Y, Takemura Y, Iwamoto K, Higashino T, and Miyashita A (1998) Carboncarbon bond cleavage of $\alpha$-hydroxybenzylheteroarenes catalyzed by cyanide ion: retro-benzoin condensation affords ketones and heteroarenes and benzyl migration affords benzylheteroarenes and arenecarbaldehydes. Chem Pharm Bull (Tokyo) 46: 199-206.

Tanabe K (2017) Microtubule depolymerization by kinase inhibitors: unexpected findings of dual inhibitors. Int $\mathrm{J} \mathrm{Mol} \mathrm{Sci} \mathbf{1 8}$.

Tulu US, Fagerstrom C, Ferentz NP, and Wandsworth P (2006) Molecular requirements for kinetochore-associated microtubule formation in mammalian cells. Curr Biol 16:536-541.

Woodruff JB, Wueseke O, and Hyman AA (2014) Pericentriolar material structure and dynamics. Philos Trans $R$ Soc Lond B Biol Sci 369:20130459.

Yao R, Kondoh Y, Natsume Y, Yamanaka H, Inoue M, Toki H, Takagi R, Shimizu T, Yamori T, Osada H, et al. (2014) A small compound targeting TACC3 revealed its different spatiotemporal contributions for spindle assembly in cancer cells. Oncogene 33:4242-4252.

Address correspondence to: ${ }^{1}$ Akira Asai, Center for Drug Discovery, Graduate School of Pharmaceutical Sciences, University of Shizuoka, 52-1 Yada, Suruga-ku, Shizuoka 422-8526, Japan. E-mail: aasai@u-shizuokaken.ac.jp; or ${ }^{2}$ Yumiko Suzuki, Department of Material and Life Sciences, Faculty of Science and Technology, Sophia University, 7-1 Kioicho, Chiyodaku, Tokyo 102-8554, Japan. E-mail: yumiko_suzuki@sophia.ac.jp 